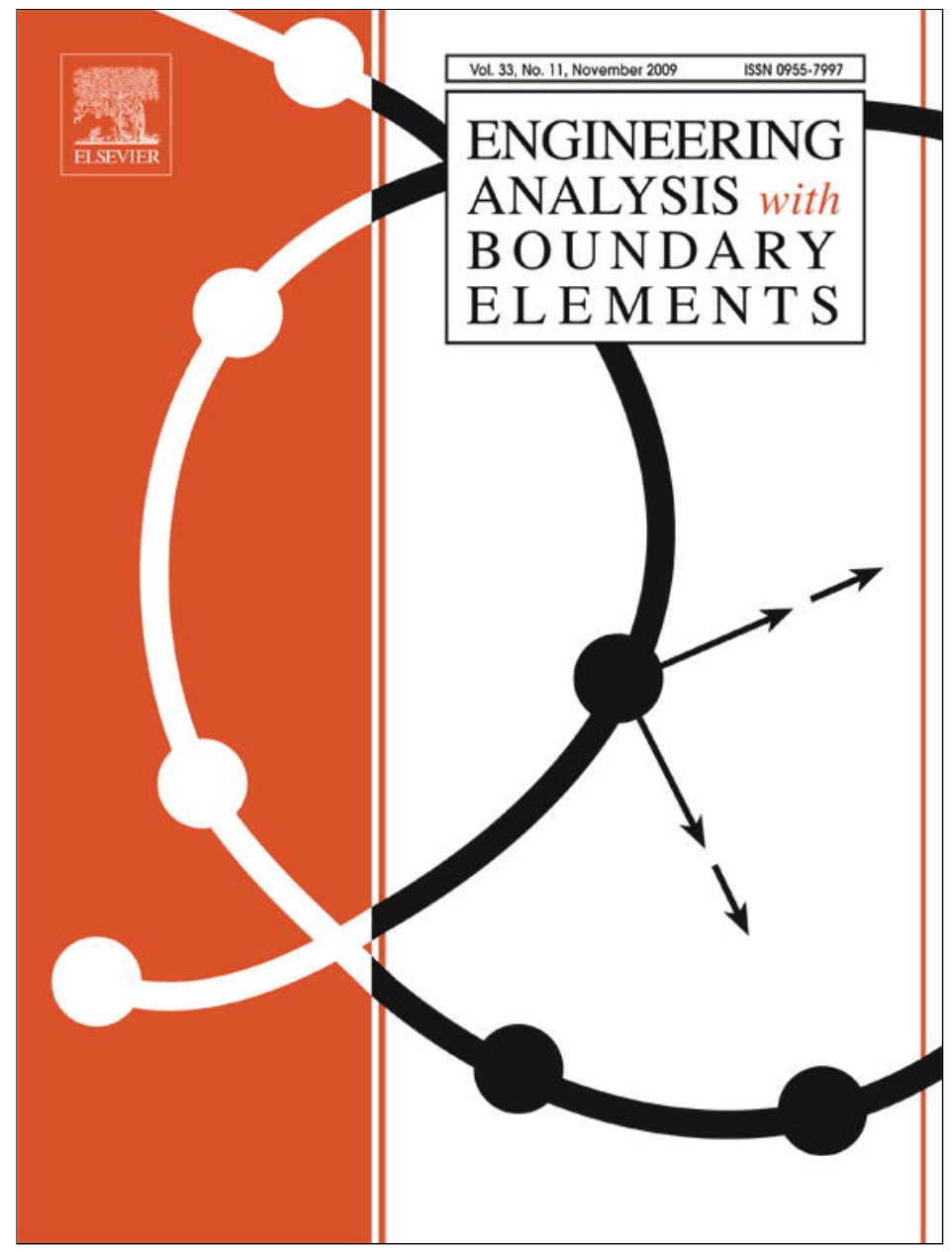

This article appeared in a journal published by Elsevier. The attached copy is furnished to the author for internal non-commercial research and education use, including for instruction at the authors institution and sharing with colleagues.

Other uses, including reproduction and distribution, or selling or licensing copies, or posting to personal, institutional or third party websites are prohibited.

In most cases authors are permitted to post their version of the article (e.g. in Word or Tex form) to their personal website or institutional repository. Authors requiring further information regarding Elsevier's archiving and manuscript policies are encouraged to visit:

http://www.elsevier.com/copyright 


\title{
Fictitious frequency revisited
}

\author{
J.T. Chen ${ }^{a, b, *}$, S.R. Lin ${ }^{c}$, J.J. Tsai ${ }^{a}$ \\ ${ }^{a}$ Department of Harbor and River Engineering, National Taiwan Ocean University, P.O. Box 7-59, Keelung 20224, Taiwan \\ ${ }^{\mathrm{b}}$ Department of Mechanical and Mechatronic Engineering, National Taiwan Ocean University, Keelung, Taiwan \\ ${ }^{\mathrm{c}}$ Institute of Nuclear Energy Research, Lungtan, Taiwan
}

\section{A R T I C L E I N F O}

\section{Article history:}

Received 10 December 2008

Accepted 9 June 2009

Available online 16 July 2009

\section{Keywords:}

Fictitious frequency

Boundary element method

Singular value decomposition

Exterior acoustics

Nonuniqueness

\begin{abstract}
A B S T R A C T
The nonexistence and nonuniqueness problems associated with integral equation methods for exterior acoustics are revisited. The Fredholm alternative theorem in conjunction with the singular value decomposition updating technique is used to simultaneously determine the fictitious frequencies and corresponding modes in exterior acoustics. After selecting the combined Helmholtz interior integral equation formulation (CHIEF) points, the influence row vectors are obtained. A criterion in selecting the minimum number of CHIEF points and their positions is proposed by testing the orthogonal condition between the influence row vector and the right unitary vector. It is proved in the discrete system for arbitrary-shape problems that the source of numerical instability of irregular frequency originates from the zero divided by zero using the generalized coordinates of unitary vectors. The mathematical structures of the four influence matrices in the dual boundary element method (BEM) are examined by using the left and right unitary matrices. Extracting the true eigenvalue and filtering out the fictitious frequency can be unified by using the updating term and updating document, respectively. Radiation problems of a cylinder and a square rod are demonstrated to see the validity of the present formulation. (c) 2009 Elsevier Ltd. All rights reserved.
\end{abstract}

\section{Introduction}

Integral equations and their implementation to boundary element method (BEM) have been employed to solve radiation and scattering problems for many years. It is well known that fictitious frequency [1,2] and spurious eigenvalue [3] appear in the exterior and interior acoustics, respectively. A good review article on this topic can be found in [4]. Both problems stem from the rank deficiency of the matrix. The Fredholm alternative theorem and singular value decomposition (SVD) updating techniques have been utilized to study the rank-deficiency matrices [5]. Juhl [6] and Poulin [7] also employed the SVD technique to study the numerical instability due to the irregular frequency. To deal with this problem, Burton and Miller [1] solved the problem by combining singular and hypersingular equations with an imaginary number. However, the calculation for the hypersingular integral is required. To avoid this computation, an alternative method was proposed by Schenck [2]. He proposed the combined Helmholtz interior integral equation formulation (CHIEF) method by collocating the point outside the domain as an auxiliary constraint to promote the rank of influence matrices. Chen et al. $[8,9]$ extended the CHIEF method to combined Helmholtz exterior integral equation for-

\footnotetext{
* Corresponding author at: Department of Harbor and River Engineering, National Taiwan Ocean University, P.O. Box 7-59, Keelung 20224, Taiwan. Tel.: +886224622192 6177; fax: +886224632375.

E-mail address: jtchen@mail.ntou.edu.tw (J.T. Chen).
}

mulation (CHEEF) method for overcoming the problem of spurious eigenvalues in the eigenproblem. However, both CHIEF and CHEEF ideas may cause failure in selecting the point. $\mathrm{Wu}$ et al. [10] proposed a CHIEF-block idea to enforce the constraints in a weighted residual sense over a small region. If the CHIEF point locates on or near the nodal line of its corresponding mode, it may not provide a valid constraint [8,9]. To overcome this problem, Chen et al. [8,9] presented an analytical study to select the valid number and position of CHIEF points for the circular case by using circulants. However, their discussions were limited in a circle case. The extension to the problems of arbitrary shapes is not trivial.

In this paper, a general case will be considered to construct a criterion for selecting the number and location of CHIEF points by employing the Fredholm alternative theorem and the SVD updating techniques. The mathematical structure for the four influence matrices in the dual BEM will be examined by using the SVD technique. Extracting the true eigenvalue and filtering out the fictitious frequency can be unified by using the SVD updating term and document, respectively. Numerical examples will be demonstrated to see the validity of the present formulation.

\section{Problem statement and review of the combined Helmholtz interior equation formulation method using the dual formulation}

In this section, the CHIEF method for the two-dimensional Helmholtz equation is briefly summarized. The governing equation 
for the exterior acoustics is

$\nabla^{2} u(x)+k^{2} u(x)=0, \quad x \in D$,

where $u(x)$ and $k$ are the acoustic pressure and the wave number, respectively, and $D$ is the exterior domain. To solve the problem by using the boundary integral formulation, the singular integral equation yields

$\pi u(x)=C P V \int_{B} T(s, x) u(s) d B(s)-R P V \int_{B} U(s, x) \frac{\partial u(s)}{\partial n_{s}} d B(s), x \in B$,

where $U(s, x)$ is the fundamental solution, $B$ is the boundary, $T(s, x)=\partial U(s, x) / \partial n_{s}, x$ is the boundary point, $s$ is the source point, $n_{s}$ is the normal vector for the source point $s, C P V$ and $R P V$ denote the Cauchy principal value and Riemann principal value, respectively. Discretization of the boundary integral equation (BIE) in Eq. (2) into $N$ constant elements yields the following linear algebraic equation:

$[U]\{t\}=[T]\{u\}$,

where $t=\partial u / \partial n,[U]$ and $[T]$ are the influence matrices.

For the fictitious frequency case, the influence matrices, $[U]$ and $[T]$, are both singular, i.e., the rank is deficient. In order to promote the rank, the CHIEF method by collocating the point outside the domain for the null-field BIE as an auxiliary constraint is applied to deal with this problem. The null-field integral equation is

$0=\int_{B} T(s, x) u(s) d B(s)-\int_{B} U(s, x) \frac{\partial u(s)}{\partial n_{s}} d B(s)$,

where $x$ is collocated outside the domain. The additional constraints are

$\langle w\rangle\{t\}=\langle v\rangle\{u\}$,

where $\langle w\rangle$ and $\langle v\rangle$ are the influence row vectors by collocating the points in the null-field singular integral equation. By combining Eq. (3) with Eq. (5), the over-determined system is obtained as

$\left[\begin{array}{c}{[U]} \\ \langle w\rangle\end{array}\right]\{t\}=\left[\begin{array}{c}{[T]} \\ \langle v\rangle\end{array}\right]\{u\}$,

if the sufficient number of CHIEF points are provided.

Similarly, the CHIEF method can be applied to the following hypersingular formulation:

$\pi \frac{\partial u(x)}{\partial n_{x}}=H P V \int_{B} M(s, x) u(s) d B(s)-C P V \int_{B} L(s, x) \frac{\partial u(s)}{\partial n_{s}} d B(s), \quad x \in B$,

where the two kernels are

$L(s, x) \equiv \frac{\partial U(s, x)}{\partial n_{x}}$,

$M(s, x) \equiv \frac{\partial^{2} U(s, x)}{\partial n_{x} \partial n_{s}}$,

and $H P V$ is the Hadamard (Mangler) principal value. By discretizing the hypersingular formulation in Eq. (7) into $N$ constant elements, the linear algebraic equation is obtained as

$[M]\{t\}=[L]\{u\}$,

where $[M]$ and $[L]$ are the influence matrices. For the case of fictitious frequency, the influence matrices, $[M]$ and $[L]$ are both singular, i.e., the rank is deficient. In order to promote the rank, the CHIEF method by collocating the point outside the domain for the null-field BIE as an auxiliary constraint, was successfully applied to deal with this problem. The null-field integral equation

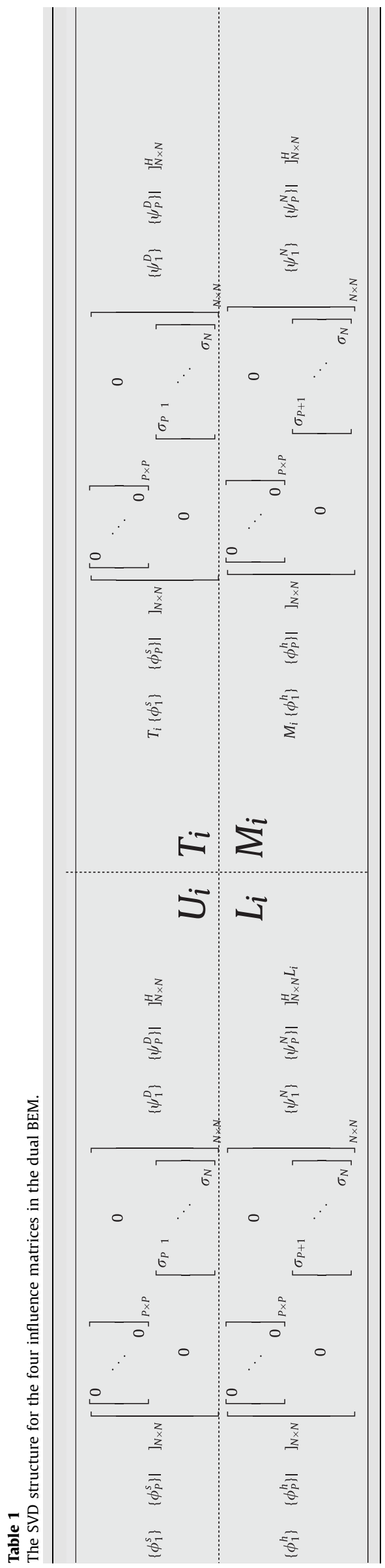






Fig. 1. The flowchart of a criterion to check the validity of the selected CHIEF point.

is obtained as

$0=\int_{B} M(s, x) u(s) d B(s)-\int_{B} L(s, x) \frac{\partial u(s)}{\partial n_{s}} d B(s)$

where $x$ is collocated outside the domain. The additional constraints are

$\left\langle w^{h}\right\rangle\{t\}=\left\langle v^{h}\right\rangle\{u\}$,

where $\left\langle w^{h}\right\rangle$ and $\left\langle v^{h}\right\rangle$ are the influence row vectors by collocating the points in the null-field hypersingular integral equation. By combining Eq. (12) with Eq. (10), an over-determined system for the conventional CHIEF concept is obtained as

$\left[\begin{array}{c}{[M]} \\ \left\langle w^{h}\right\rangle\end{array}\right]\{t\}=\left[\begin{array}{c}{[L]} \\ \left\langle v^{h}\right\rangle\end{array}\right]\{u\}$

if the sufficient number of CHIEF points are provided. 

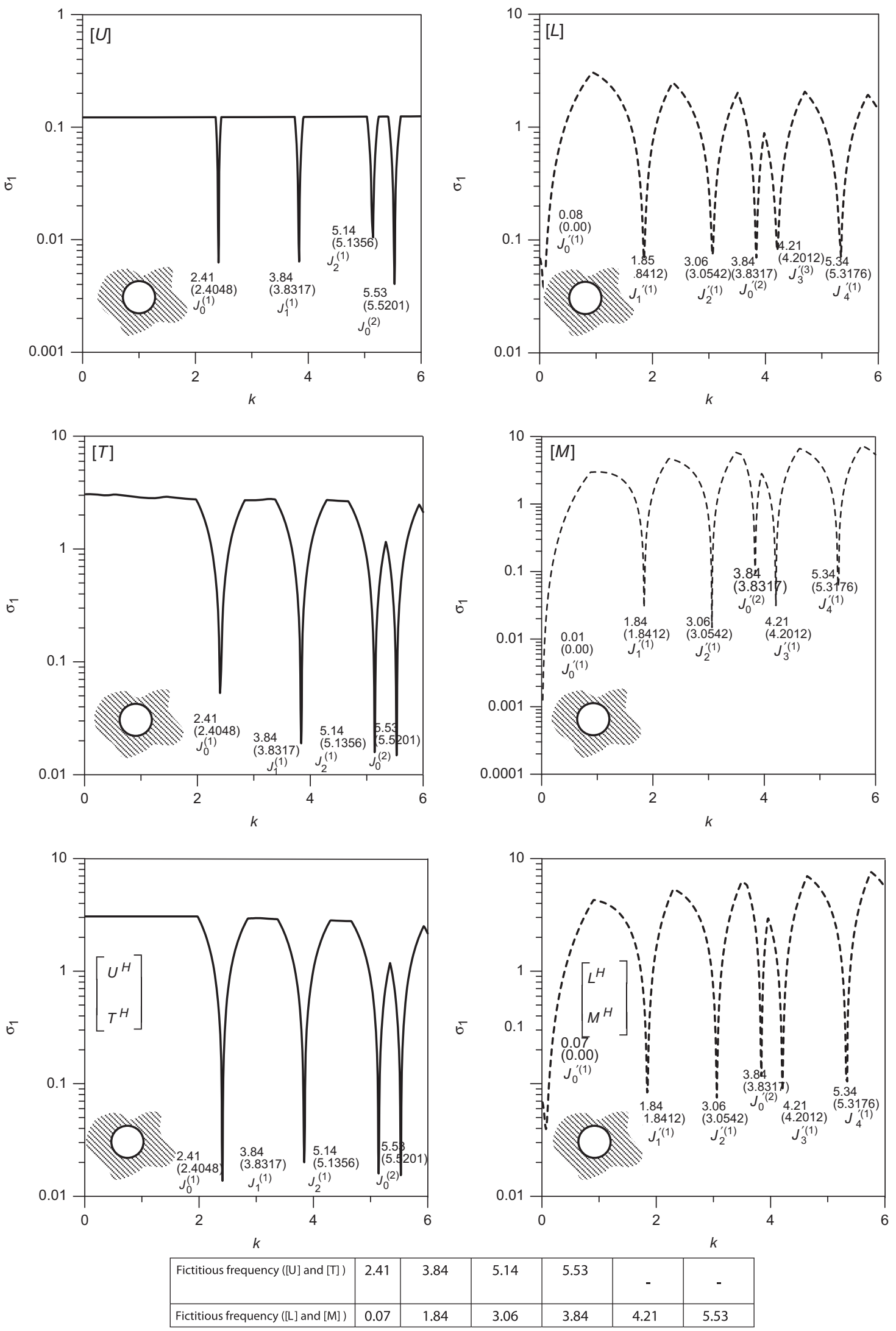

Fig. 2. The first minimum singular value versus the wave number $k$ by using SVD updating technique. 
3. Detection of the fictitious frequency and fictitious mode for exterior acoustics using the Fredholm alternative and the updating techniques of singular value decomposition

Fredholm alternative theorem. The linear algebraic equation $[K]\{u\}=\{b\}$ has a unique solution if and only if the continuous solution to the homogeneous equation

$[K]\{u\}=\{0\}$

is $\{u\} \equiv\{0\}$. Alternatively, the homogeneous equation has at least one solution if the homogeneous adjoint equation

$[K]^{H}\{\phi\}=\{0\}$

has a nontrivial solution $\{\phi\}$, where $[K]^{H}$ is the transpose conjugate matrix of $[K]$ and $\{b\}$ must satisfy the constraint $\left(\{b\}^{H}\{\phi\}=0\right)$. The UT formulation of Eq. (3) yields

$[U(k)]\{t\}=[T(k)]\{u\}=\{b\}$.

According to the Fredholm alternative theorem, Eq. (16) has at least one solution for $\{t\}$ if the homogeneous adjoint equation

$\left[U\left(k_{f}\right)\right]^{H}\left\{\phi_{1}\right\}=\{0\}$,

has a nontrivial solution $\left\{\phi_{1}\right\}$, where $k_{f}$ is the fictitious wave number, and in which the constraint $\left(\{b\}^{H}\left\{\phi_{1}\right\}=0\right)$ must be satisfied. Substitution of $\{b\}=\left[T\left(k_{f}\right)\right]\{u\}$ for the Dirichlet problem in Eq. (16) into $\{b\}^{H}\left\{\phi_{1}\right\}=0$ yields

$\{u\}^{H}\left[T\left(k_{f}\right)\right]^{H}\left\{\phi_{1}\right\}=0$.

Eq. (18) implies

$\left[T\left(k_{f}\right)\right]^{H}\left\{\phi_{1}\right\}=\{0\}$

since $\{u\}$ is an arbitrary vector, where $\left\{\phi_{1}\right\}$ is the fictitious mode. Combination of Eq. (17) with Eq. (19) yields

$\left[\begin{array}{c}{\left[U\left(k_{f}\right)\right]^{H}} \\ {\left[T\left(k_{f}\right)\right]^{H}}\end{array}\right]\left\{\phi_{1}\right\}=\{0\} \quad$ or $\left\{\phi_{1}\right\}^{H}\left[\left[U\left(k_{f}\right)\right] \quad\left[T\left(k_{f}\right)\right]\right]=\{0\}$.

Eq. (20) indicates that the two matrices have the same fictitious mode $\left\{\phi_{1}\right\}$ corresponding to the same zero singular value when

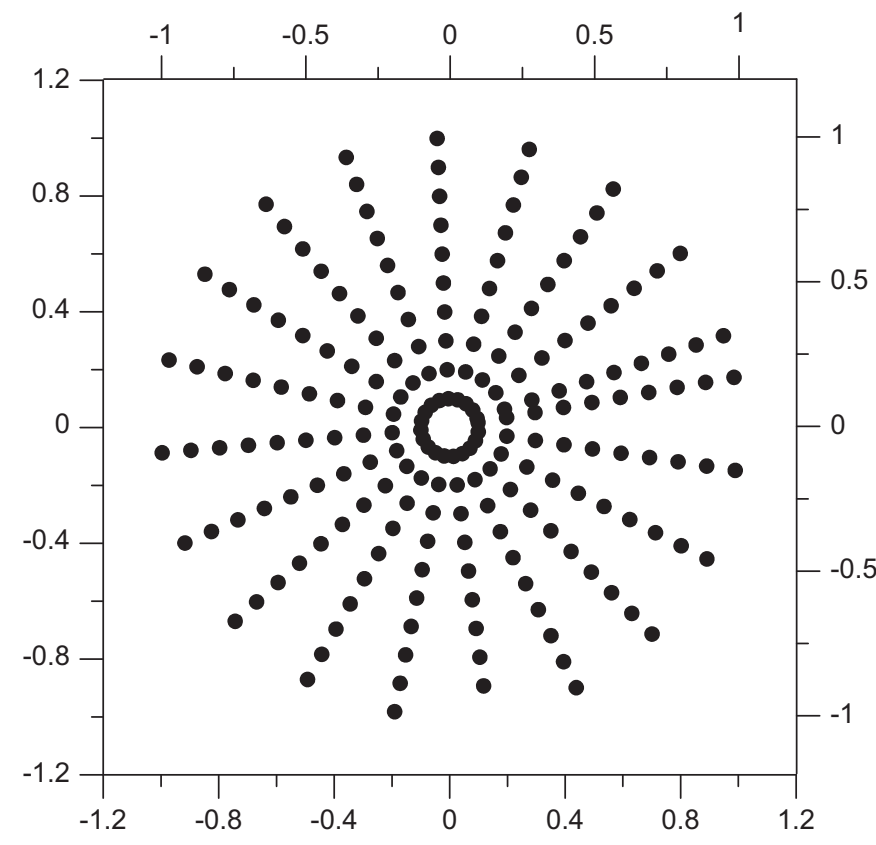

Fig. 3. CHIEF points outside the domain. the rank deficiency occurs in the case of fictitious frequency. The former and latter matrices in Eq. (20) are the expressions of updating term and of updating document, respectively. The fictitious wave number, $k_{f}$ of a multiplicity $P$, satisfies

$$
\left[\begin{array}{c}
{\left[U_{i}\left(k_{f}^{S}\right)\right]^{H}} \\
{\left[T_{i}\left(k_{f}^{s}\right)\right]^{H}}
\end{array}\right]\left\{\phi_{j}\right\}=\{0\}, \quad j=1,2, \ldots, P,
$$

$\left[\begin{array}{c}{\left[L_{i}\left(k_{f}^{h}\right)\right]^{H}} \\ {\left[M_{i}\left(k_{f}^{h}\right)\right]^{H}}\end{array}\right]\left\{\phi_{j}\right\}=\{0\}, \quad j=1,2, \ldots, P$,

where the subscript $i$ denotes the use of interior kernel for the exterior problem using null-field integral equations and $k_{f}^{s}$ and $k_{f}^{h}$ are the fictitious wave number for the singular and hypersingular formulations, respectively.
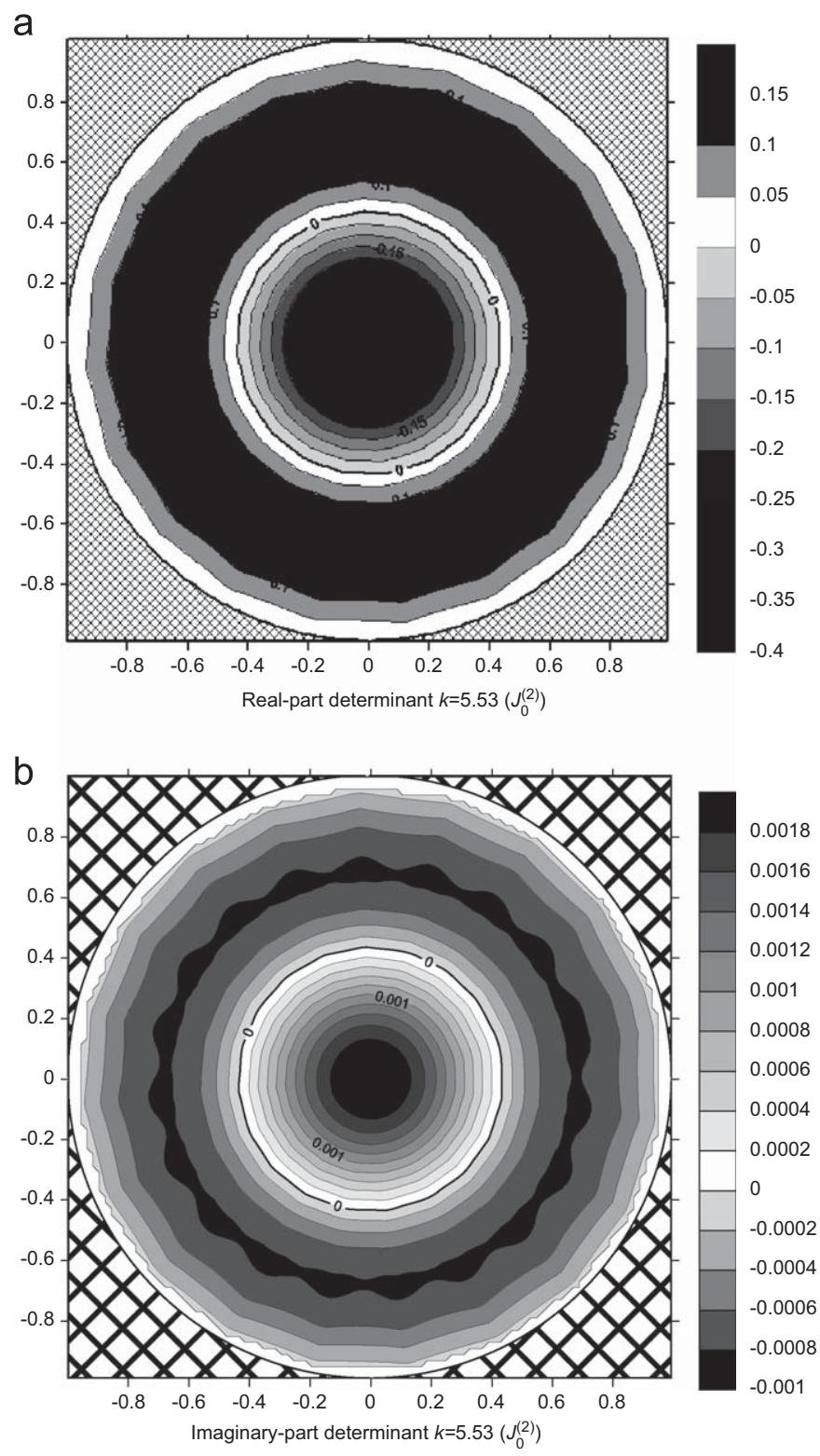

Fig. 4. (a) The real-part determinant for the fictitious frequency with multiplicity of one $(P=1)$. (b) The imaginary-part determinant for the fictitious frequency with multiplicity of one $(P=1)$. 


\section{Mathematical structure for the four influence matrices} using the updating technique of singular value decomposition

According to the SVD technique, Eq. (21) results in

$\left[U_{i}\right]\left\{\psi_{j}^{(U)}\right\}=0\left\{\phi_{j}\right\}=\{0\}, \quad j=1,2, \ldots, P$,

$\left[T_{i}\right]\left\{\psi_{j}^{(T)}\right\}=0\left\{\phi_{j}\right\}=\{0\}, \quad j=1,2, \ldots, P$,

where $\left\{\psi_{j}^{(U)}\right\}$ and $\left\{\psi_{j}^{(T)}\right\}$ are the right unitary vectors for $[U]$ and $[T]$, $\left\{\phi_{j}\right\}$ are the common left unitary vector. For the eigenproblem, the singular $U T$ and the hypersingular $L M$ formulations can obtain the same eigenmode $\psi^{D}$, where $\left\{\psi^{D}\right\}$ is the boundary mode for the Dirichlet eigenproblem. By using the updating term for deriving the true boundary mode $\left\{\psi^{D}\right\}$, an assembled system is obtained as

$\left[\begin{array}{c}{\left[U_{e}\right]} \\ {\left[L_{e}\right]}\end{array}\right]\left\{\psi_{j}^{D}\right\}=\{0\}, \quad j=1,2, \ldots, P$,

a

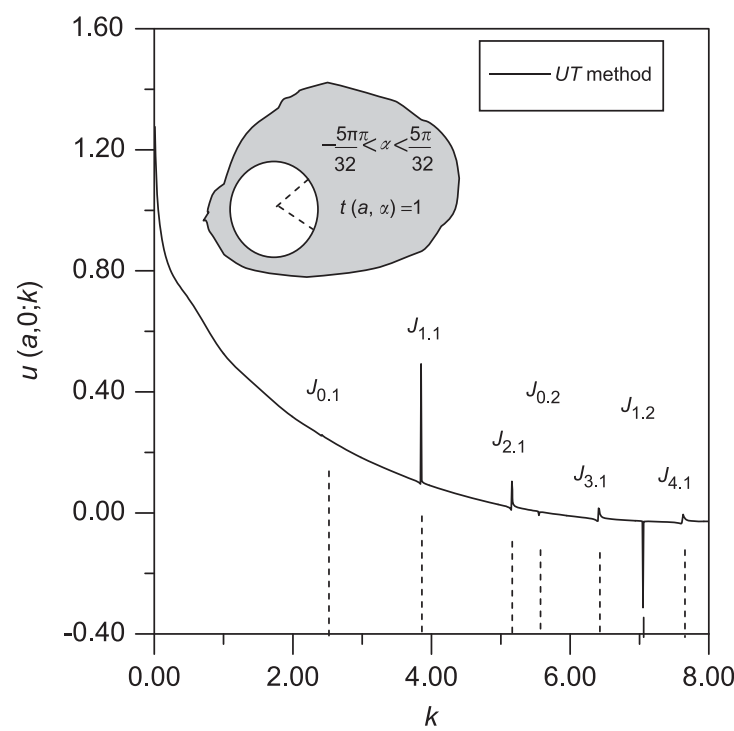

where the subscript $e$ denotes the use of exterior kernel for the interior problem using the null-field integral formulation. The kernel functions have the following symmetry and transpose symmetry properties:

$U_{e}(s, x)=U_{i}(x, s)$ or $\left[U_{e}\right]=\left[U_{i}\right]$ symmetry,

and

$L_{e}(s, x)=T_{i}(x, s) \quad$ or $\quad\left[L_{e}\right]=\left[T_{i}\right] \quad$ transpose symmetry

By using Eqs. (26) and (27), Eq. (25) reduces to

$\left[\begin{array}{c}{\left[U_{i}\right]} \\ {\left[T_{i}\right]}\end{array}\right]\left\{\psi_{j}^{D}\right\}=\{0\}, \quad j=1,2, \ldots, P$.

Comparison of Eq. (28) with Eqs. (23) and (24) yields

$\left\{\psi_{j}^{(U)}\right\}=\left\{\psi_{j}^{(T)}\right\}=\left\{\psi_{j}^{D}\right\}, \quad j=1,2, \ldots, P$.

b

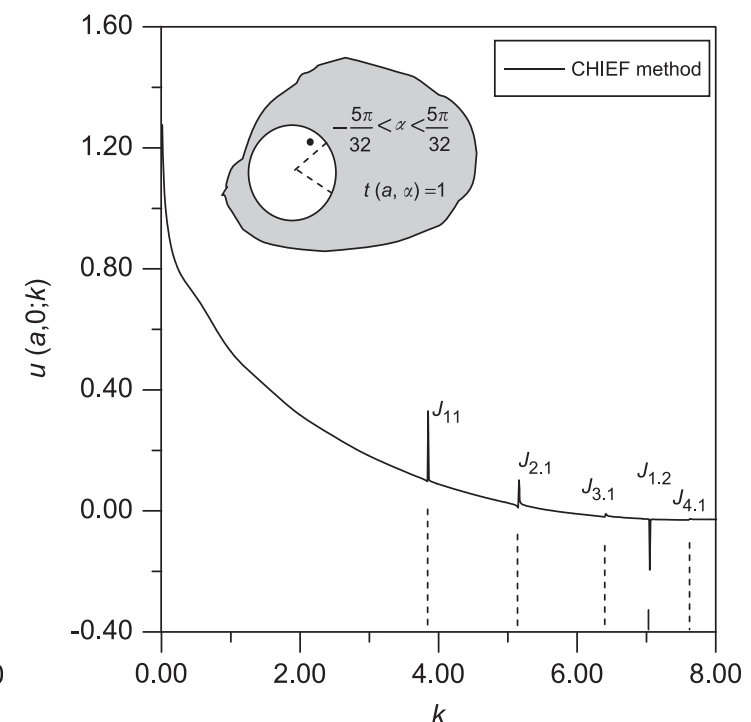

Fig. 5. (a) The boundary value versus the wave number $k$ by using the $U T$ formulations. (b) The boundary value versus the wave number $k$ by locating one CHIEF point away from the nodal line.

a



Real-parp determinant

$k=3.84\left(J_{1}^{(1)}\right)$ b

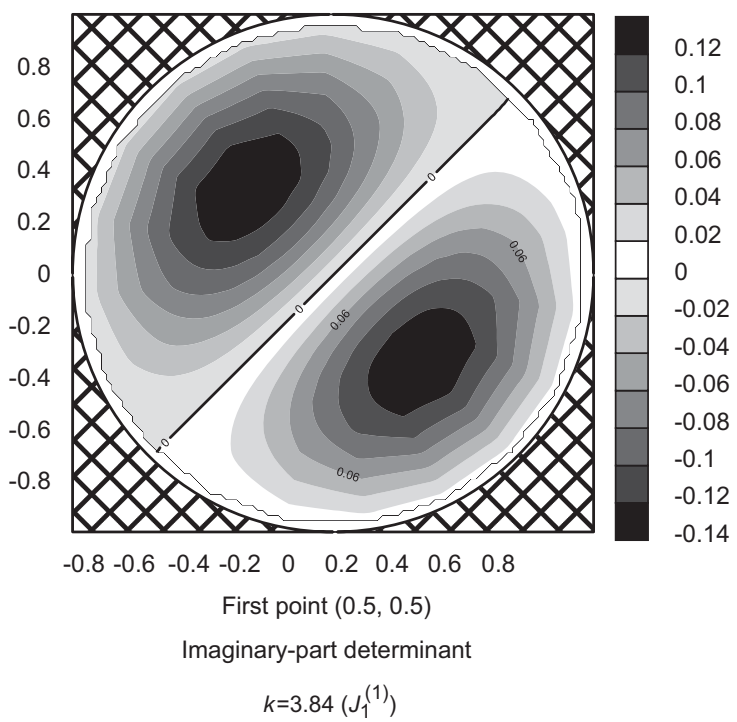

Fig. 6. (a) The real-part determinant for the fictitious frequency with multiplicity of two $(P=2)$. (b) The imaginary-part determinant for the fictitious frequency with multiplicity of two $(P=2)$. 
It means that the $\left[U_{i}\right]$ and $\left[T_{i}\right]$ matrices for the exterior acoustics, have the same right singular vector $\left(\left\{\psi_{j}^{D}\right\}\right)$ as $\left[U_{e}\right]$ and $\left[L_{e}\right]$ matrices have for the Dirichlet eigenproblem.

In order to examine the left and right singular vectors in the singular matrix, Eq. (21) is rewritten as follows:

$\left[\begin{array}{l}{\left[U_{i}\left(k_{f}\right)\right]^{H}} \\ {\left[T_{i}\left(k_{f}\right)\right]^{H}}\end{array}\right]_{2 N \times N}\left\{\phi_{j}\right\}_{N \times 1}=0\left\{\begin{array}{l}\psi_{j}^{D} \\ \psi_{j}^{D}\end{array}\right\}_{2 N \times 1}, j=1,2, \ldots, P$.

Generally speaking, the matrix of Eq. (30) can be decomposed into

$\left[\begin{array}{c}{\left[U_{i}\right]^{H}} \\ {\left[T_{i}\right]^{H}}\end{array}\right]_{2 N \times N}=\left[\Psi^{D}\right]_{2 N \times 2 N}[\Sigma]_{2 N \times N}[\Phi]_{N \times N}^{H}$,

where

$\left[\Psi^{D}\right]_{2 N \times 2 N}=\left[\begin{array}{lllllll}\left\{\psi_{1}^{D}\right\} & \cdots & \left\{\psi_{P}^{D}\right\} & \vdots & \left\{\psi_{P+1}\right\} & \cdots & \left\{\psi_{2 N}\right\} \\ \left\{\psi_{1}^{D}\right\} & \cdots & \left\{\psi_{P}^{D}\right\} & \vdots & \left\{\psi_{P+1}\right\} & \cdots & \left\{\psi_{2 N}\right\}\end{array}\right]_{2 N \times 2 N}$,

$[\Sigma]_{2 N \times N}=\left[\begin{array}{ccccccc}0 & \cdots & \cdots & \cdots & \cdots & \cdots & 0 \\ \vdots & 0 & & & & & \vdots \\ \vdots & & \ddots & & & & \vdots \\ \vdots & & & 0 & & & \vdots \\ \vdots & & & & \sigma_{p+1} & & \vdots \\ \vdots & & & & & \ddots & \vdots \\ 0 & \cdots & \cdots & \cdots & \cdots & \cdots & \sigma_{N} \\ 0 & \cdots & \cdots & \cdots & \cdots & \cdots & 0 \\ \vdots & \ddots & & & & & \vdots \\ \vdots & & \ddots & & & & \vdots \\ \vdots & & & \ddots & & & \vdots \\ \vdots & & & & \ddots & & \vdots \\ \vdots & & & & & \ddots & \vdots \\ \vdots & \cdots & \cdots & \cdots & \cdots & \cdots & 0\end{array}\right]_{2 N \times N}$

and

$[\Phi]_{N \times N}^{H}=\left[\begin{array}{lllllll}\left\{\phi_{1}\right\} & \cdots & \left\{\phi_{P}\right\} & \mid & \left\{\phi_{P+1}\right\} & \cdots & \left\{\phi_{N}\right\}\end{array}\right]_{N \times N}^{H}$.

Eq. (31) indicates that all the fictitious modes $\left\{\phi_{i}\right\}, 1 \leq i \leq P$, and the true modes $\left\{\psi_{i}^{D}\right\}, 1 \leq i \leq P$, are simultaneously obtained once the updating matrix is decomposed by using the SVD updating technique.

In summary, the SVD structure for the four influence matrices in the dual BEM is unified in Table 1.

\section{Source of numerical instability_zero divided by zero}

The analytical study and numerical experiments for the optimum numbers and proper positions of the selected CHIEF points have been proposed by Chen et al. for a circular case. Here, the general case in a discrete system is extended. In the case of the fictitious frequency of multiplicity $P, P$ CHIEF points are needed. One can obtain $P$ fictitious modes by using Eq. (21). The source of numerical instability stems from zero divided by zero. By employing the right unitary vectors $\left\{\psi_{i}\right\}$ embedded in the [T] and $[U]$ matrices, the boundary data are expanded into

$\{u\}=\sum_{i=1}^{N} \beta_{i}\left\{\psi_{i}^{(T)}\right\}=\left[\Psi^{(T)}\right]\{\beta\}$,

$\{t\}=\sum_{i=1}^{N} \alpha_{i}\left\{\psi_{i}^{(U)}\right\}=\left[\Psi^{(U)}\right]\{\alpha\}$,

where $N$ is the number of unknowns, $\alpha_{i}$ and $\beta_{i}$ are the generalized coordinates for $u$ and $t$, respectively. By using the SVD technique,
Eq. (16) is rewritten as

$\left[\Phi^{(U)}\right]\left[\Sigma^{(U)}\right]\{\alpha\}=\{b\}$.

By premultiplying the regular mode $\left\{\phi_{i}^{(U)}\right\}^{H}, P+1 \leq i \leq N$, to both sides of Eq. (37) and using the orthogonal property, Eq. (37) is changed to

$\sigma_{i}^{(U)} \alpha_{i}=\left\{\phi_{i}^{(U)}\right\}^{H}\{b\}, \quad P+1 \leq i \leq N$.

Since the singular values $\sigma_{i}^{(U)}, P+1 \leq i \leq N$, are nonzero, the generalized coordinates $\alpha_{i}, P+1 \leq i \leq N$, can be easily

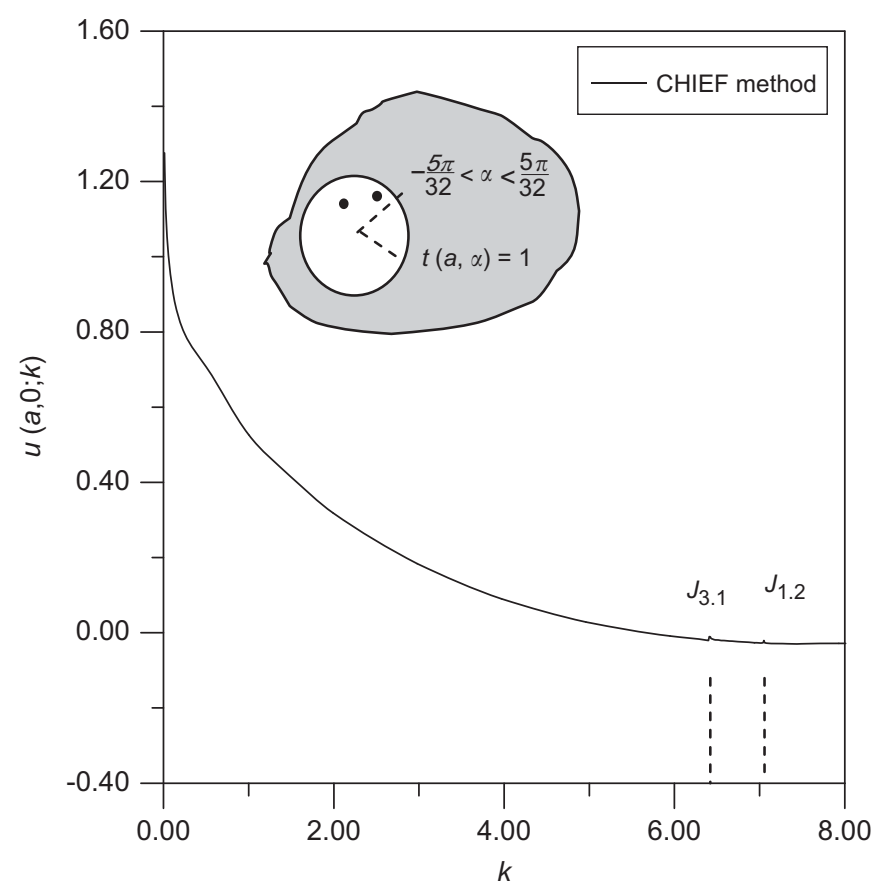

Fig. 7. The boundary value versus the wave number $k$ by locating two CHIEF points on the nodal line.

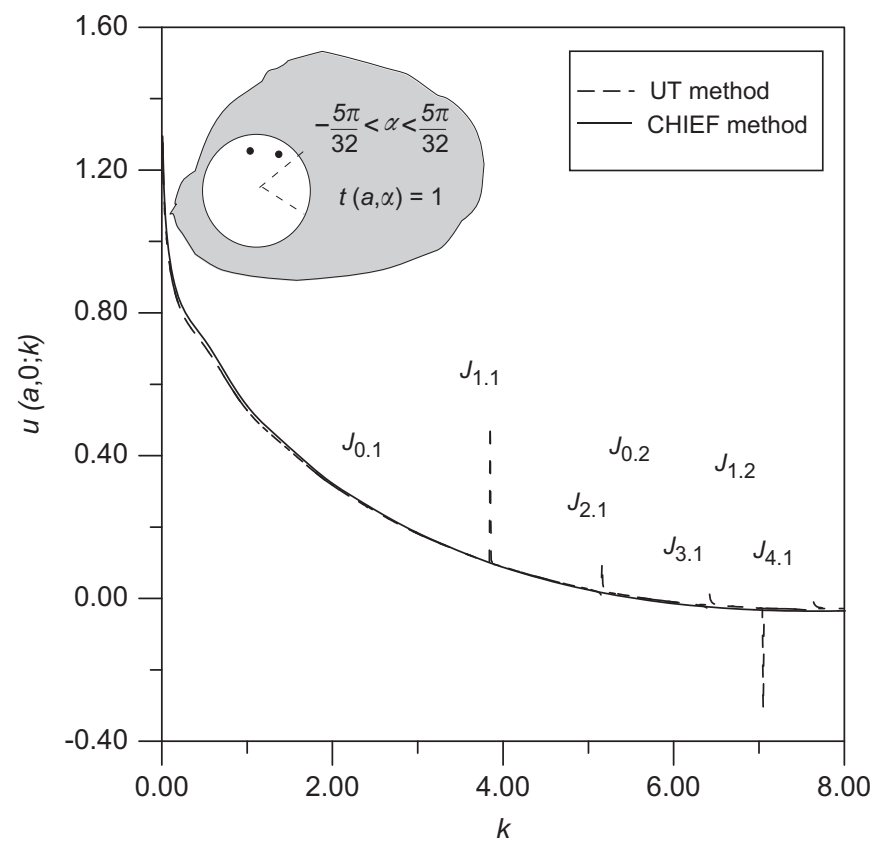

Fig. 8. The boundary value versus the wave number $k$ by locating two CHIEF points away from the nodal line. 



\begin{tabular}{|l|l|l|l|l|l|}
\hline Fictitious frequency ([U] and [T] ) & 2.22 & 3.51 & 4.44 & 4.96 & 5.66 \\
\hline Fictitious frequency ([L] and [M] ) & 0.07 & 1.57 & 2.22 & 3.14 & 3.51 \\
\hline
\end{tabular}

Fig. 9. The first minimum singular value versus the wave number $k$ by using SVD updating technique. 
determined by

$\alpha_{i}=\frac{1}{\sigma_{i}^{(U)}}\left\{\phi_{i}^{(U)}\right\}^{H}\{b\}, \quad P+1 \leq i \leq N$.

By premultiplying the fictitious mode $\left\{\phi_{i}^{(U)}\right\}^{H}, 1 \leq i \leq P$, to both sides of Eq. (37), it is reduced to

$\sigma_{i}^{(U)} \alpha_{i}=\left\{\phi_{i}^{(U)}\right\}^{H}\{b\}, \quad 1 \leq i \leq P$.

Since the singular values $\sigma_{i}^{(U)}, 1 \leq i \leq P$, are zero, the coefficients $\alpha_{i}, 1 \leq i \leq P$, cannot be determined due to zero divided by zero from Eq. (39) in the fictitious frequency of multiplicity $P$.

It is interesting to find that the generalized coordinates, $\alpha_{1}, \alpha_{2}, \ldots$, and $\alpha_{P}$ are the terms of zero divided by zero in Eq. (40) since

$\left\{\phi_{i}^{(U)}\right\}^{H}[T]\{\bar{u}\}=0, \quad P+1 \leq i \leq N$,

after using $\{b\}=[T]\{\bar{u}\}$ and $[T]^{H}\left\{\phi_{i}^{(U)}\right\}=\{0\}$ from Eq. (19).

\section{A criterion to check the validity of CHIEF points}

For the fictitious frequency of a multiplicity $P$, the generalized coordinates $\alpha_{1}, \alpha_{2}, \ldots, \alpha_{P-1}$ and $\alpha_{P}$ cannot be determined from Eq. (39). By choosing $P$ CHIEF points, additional constraints are obtained as

$\left[\begin{array}{lll}U_{P P} & \vdots & U_{P K}\end{array}\right]\left\{\begin{array}{c}\alpha_{1} \\ \alpha_{2} \\ \vdots \\ \alpha_{P} \\ --- \\ \alpha_{P+1} \\ \vdots \\ \alpha_{N}\end{array}\right\}=\left[\begin{array}{lll}T_{P P} & \vdots & T_{P K}\end{array}\right]\{\beta\}$,

where the subscripts $P$ and $K$ denote the degree of freedom separated by the fictitious set $(1,2, \ldots, P)$ and the regular set $(P+1, P+2, \ldots, N)$. The elements in $\left[U_{P P}\right],\left[U_{P K}\right],\left[T_{P P}\right]$ and $\left[T_{P K}\right]$ are defined as

$\left(U_{P P}\right)_{i j}=\left\langle w_{i}\right\rangle\left\{\psi_{j}^{(U)}\right\}, \quad 1 \leq i, j \leq P$,

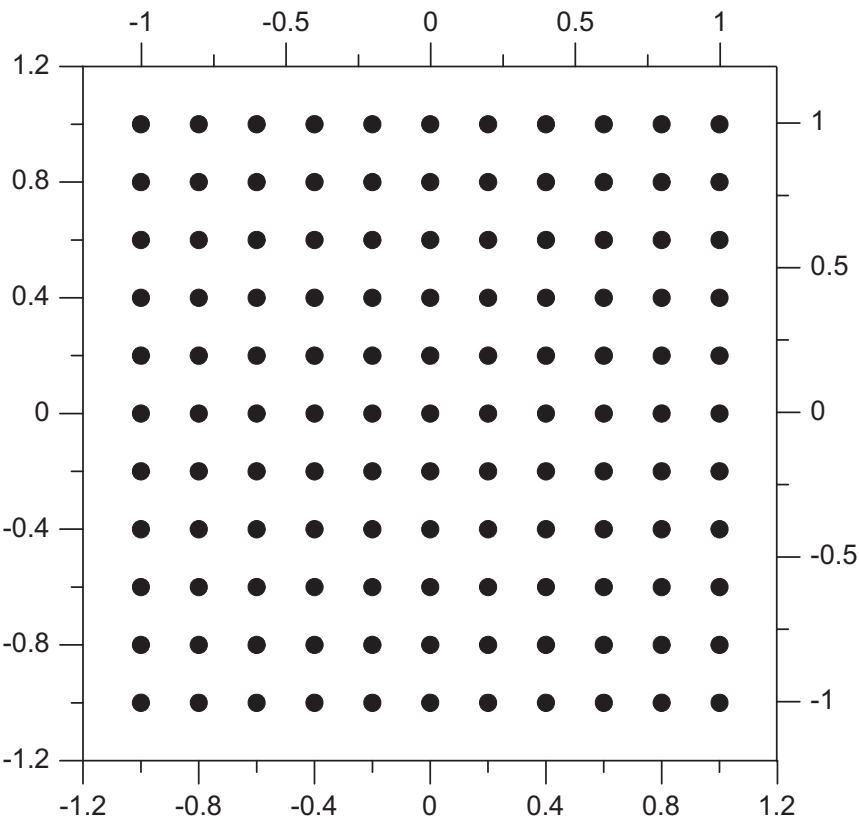

Fig. 10. CHIEF points outside the domain.

$$
\left(U_{P K}\right)_{i j}=\left\langle w_{i}\right\rangle\left\{\psi_{j}^{(U)}\right\}, \quad 1 \leq i \leq P, \quad P+1 \leq j \leq N,
$$

$\left(T_{P P}\right)_{i j}=\left\langle v_{i}\right\rangle\left\{\psi_{j}^{(T)}\right\}, \quad 1 \leq i, j \leq P$,

$\left(T_{P K}\right)_{i j}=\left\langle v_{i}\right\rangle\left\{\psi_{j}^{(T)}\right\}, \quad 1 \leq i \leq P, \quad P+1 \leq j \leq N$,

where $\left\langle v_{i}\right\rangle$ and $\left\langle w_{i}\right\rangle$ are the influence row vectors by collocating the null-field integral equations on the CHIEF points. Since $\alpha_{P+1}, \alpha_{P+2}, \ldots$, and $\alpha_{N}$ can be determined by Eq. (39), Eq. (42) reduces to

$\left[U_{P P}\right]\left\{\begin{array}{c}\alpha_{1} \\ \vdots \\ \alpha_{P}\end{array}\right\}=\left[T_{P P} \mid T_{P K}\right]\left\{\begin{array}{c}\beta_{1} \\ \vdots \\ \beta_{N}\end{array}\right\}-\left[U_{P K}\right]\left\{\begin{array}{c}\alpha_{P+1} \\ \vdots \\ \alpha_{N}\end{array}\right\}=\{f\}$.

The terms of the right hand side of the equal sign can be calculated as a load vector $\{f\}$ since their values can be determined. The unknown vector, $\{\alpha\}_{P_{\times 1}}$, is solvable once the
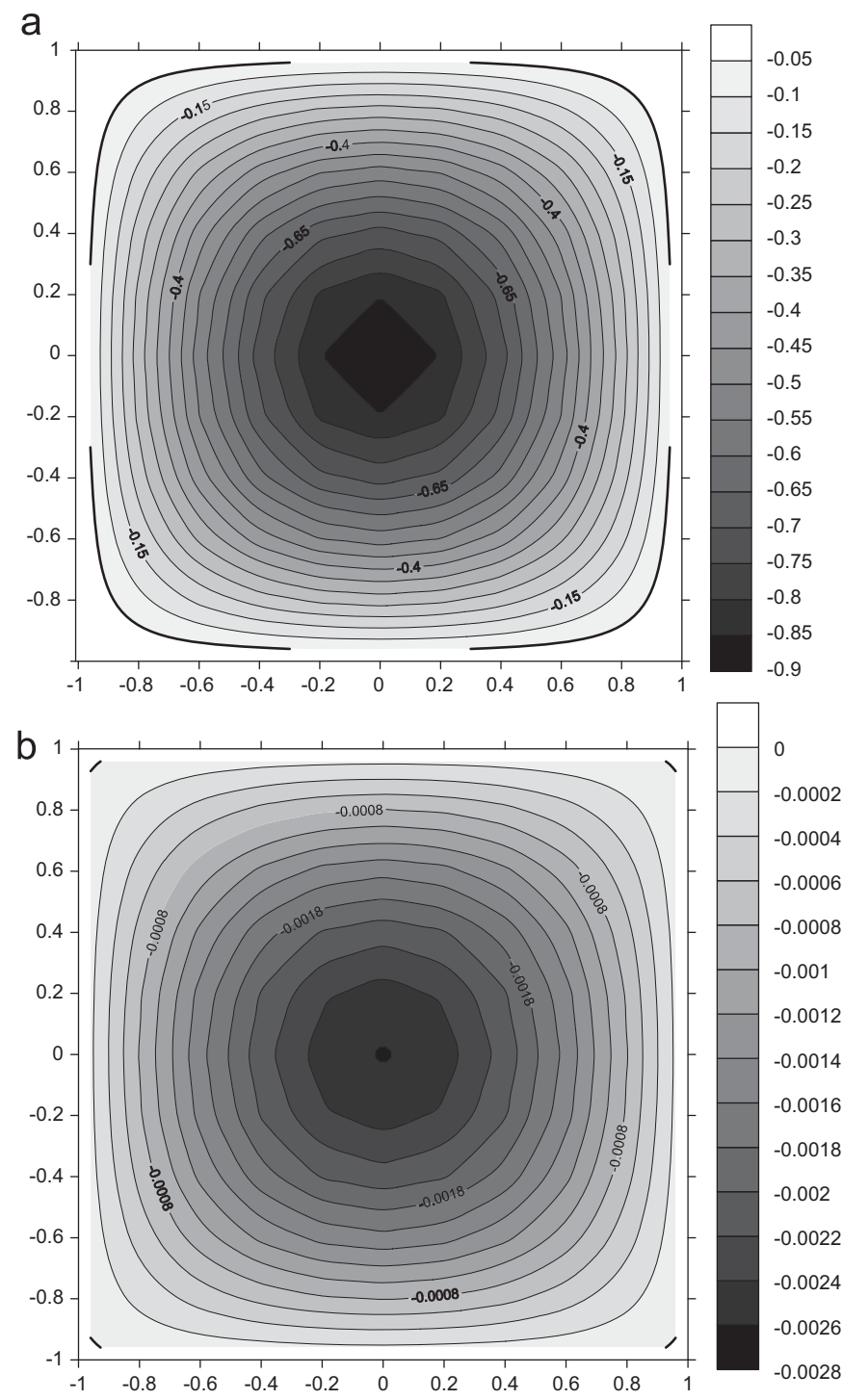

Fig. 11. (a) The real-part determinant for the fictitious frequency with multiplicity of one $(P=1), R=2.22$. (b) The imaginary-part determinant for the fictitious frequency with multiplicity of one $(P=1), R=2.22$. 
determinant of the matrix $\left[U_{P P}\right]$ is nonzero as follows:

$\operatorname{det}\left|\begin{array}{ccc}\left\langle w_{1}\right\rangle\left\{\psi_{1}^{(U)}\right\} & \cdots & \left\langle w_{1}\right\rangle\left\{\psi_{P}^{(U)}\right\} \\ \vdots & \ddots & \vdots \\ \left\langle w_{P}\right\rangle\left\{\psi_{1}^{(U)}\right\} & \cdots & \left\langle w_{P}\right\rangle\left\{\psi_{P}^{(U)}\right\}\end{array}\right| \neq 0$.

Whether the number of the CHIEF point is sufficient or not depends on the multiplicity $P$, i.e., at least $P$ CHIEF points for the fictitious frequency of multiplicity $P$ to determine the $P$ coefficients $\left(\alpha_{1}, \ldots, \alpha_{P}\right)$ in Eq. (47) are needed. Once the $P$ CHIEF points are selected, the solvability condition depends on the nonzero determinant of Eq. (48).

For the special case of multiplicity one $(P=1)$, Eq. (48) reduces to

$\left\langle w_{1}\right\rangle\left\{\psi_{1}^{(U)}\right\} \neq 0$.

By collocating the interior point, the magnitude of the determinant represents the inner product of the influence row vector and the interior mode since $\left\{\psi^{(U)}\right\}$ is the true boundary mode of the Dirichlet eigenproblem. The magnitude is equal to the amplitude of interior mode for the corresponding interior eigenvalue.

For the special case of multiplicity two $(P=2)$, Eq. (48) reduces to

$\operatorname{det}\left|\begin{array}{cc}\left\langle w_{1}\right\rangle\left\{\psi_{1}^{(U)}\right\} & \left\langle w_{1}\right\rangle\left\{\psi_{2}^{(U)}\right\} \\ \left\langle w_{2}\right\rangle\left\{\psi_{1}^{(U)}\right\} & \left\langle w_{2}\right\rangle\left\{\psi_{2}^{(U)}\right\}\end{array}\right| \neq 0$.

In the following examples, both the multiplicity one $(P=1)$ and two $(P=2)$ will be discussed for the cylinder and square rod radiators.

For the Neumann problem, the same criterion is adopted in a similar way by replacing $\langle w\rangle$ and $\left\{\psi_{j}^{(U)}\right\}$ with $\langle v\rangle$ and $\left\{\psi_{j}^{(T)}\right\}$, respectively.

\section{Numerical examples}

\subsection{Case 1: a cylinder radiator}

An exterior acoustic problem of a cylinder with radius $a=1 \mathrm{~m}$ subjected to the nonuniform radiation for the Neumann boundary conditions is considered. According to the flowchart illustrated in Fig. 1, the Fredholm alternative theorem and SVD updating techniques are employed to detect the fictitious frequencies as shown in Fig. 2. Twenty boundary elements are considered. It is found that $[U]$ and $[T]$ matrices have the same fictitious poles of $J_{n}(k a)=0,(n=0,1,2, \ldots)$, as predicted by using circulants. The spurious poles agree with the true poles of the Dirichlet eigenproblem. For the hypersingular formulation, $[L]$ and $[M]$ matrices also have the same fictitious poles of $J_{n}^{\prime}(k a)=0,(n=0,1,2, \ldots)$, which are the true eigenvalues for the Neumann problem. After checking the multiplicity of the fictitious pole, two cases of multiplicity one $\left(k=J_{0}^{(2)}=5.53\right)$, and multiplicity two $\left(k=J_{1}^{(1)}=3.84\right)$, are adopted for demonstrating the validity of the present formulation.

1. Multiplicity of one $\left(k=J_{0}^{(2)}=5.53\right)$ : For selecting all the possible CHIEF points, their positions locate inside the circle as shown in Fig. 3. In this case, the determinants of Eq. (49) were calculated for each interior point and were plotted as shown in Fig. 4(a) and (b). Contour plot shows the distribution of the magnitude of the real and imaginary parts of determinant. The selected CHIEF point of the dark color is valider than the point with the white color. The failure points are found on the nodal line with white color and the results matched well with the analytical prediction. Without locating any CHIEF points, the fictitious frequencies appear as shown in Fig. 5(a). By selecting one valid CHIEF point which is not on the nodal line, the fictitious frequencies $\left(k=J_{0}^{(1)}=2.41\right.$ and $\left.J_{0}^{(2)}=5.53\right)$ are suppressed as shown in Fig. 5(b).

2. Multiplicity of two $\left(k=J_{1}^{(1)}=3.84\right)$ : In this case, the first CHIEF point was fixed at $(0.5,0.5)$ and then consider the other CHIEF point as a variable. For selecting all the possible CHIEF points, their positions locate inside the circle as shown in Fig. 3. The determinants were calculated by changing the second position of the CHIEF point in the interior region and were plotted in Fig. 6(a) and (b). Contour plot shows the distribution of the magnitude of the real and imaginary parts of determinants. Since the unitary vector is adopted, it is found that real-part determinant is very small. The selected CHIEF point of the dark color is valider than the point with the white color. The failure points are found on the nodal line with white color and the results match well with the analytical prediction. If two improper CHIEF points are selected, the fictitious frequencies $\left(k=J_{1}^{(3)}=6.380\right)$ and $\left(k=J_{2}^{(1)}=7.061\right)$ cannot be suppressed as shown in Fig. 7. By selecting the two proper CHIEF points away from the nodal line, the fictitious frequency disappears as shown in Fig. 8.

\subsection{Case 2: plane scattering wave for a rigid square rod}

An exterior scattering problem of a square rod with a lateral length $a=2 \mathrm{~m}$ for the Neumann boundary conditions is considered here. The Fredholm alternative theorem and SVD updating a

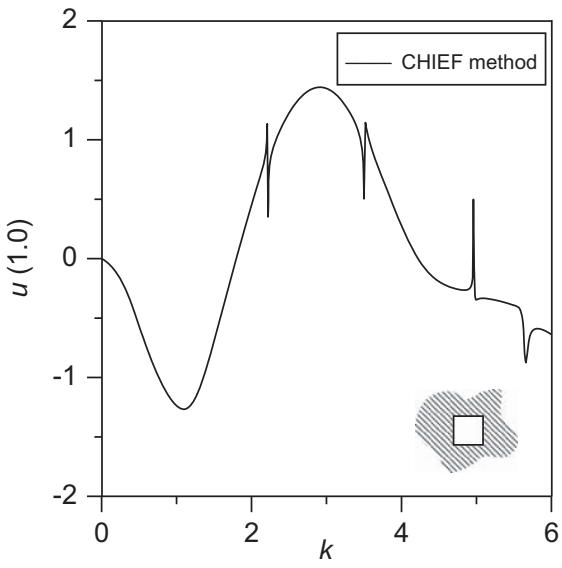

b

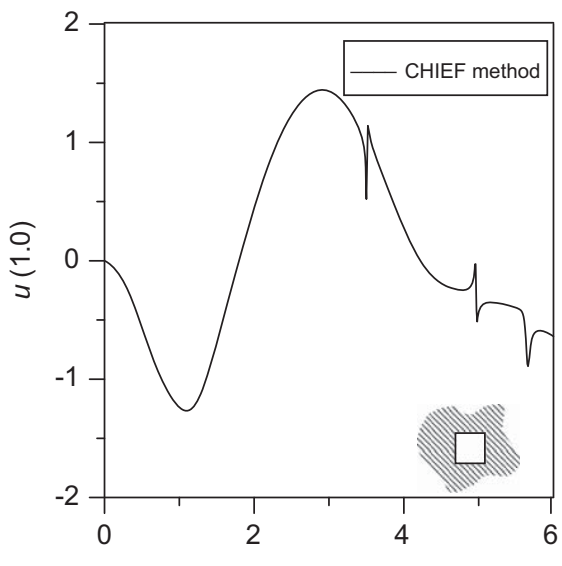

Fig. 12. (a) The boundary value versus the wave number $k$ by using the $U T$ formulations. (b) The boundary value versus the wave number $k$ by locating one CHIEF point. 
a

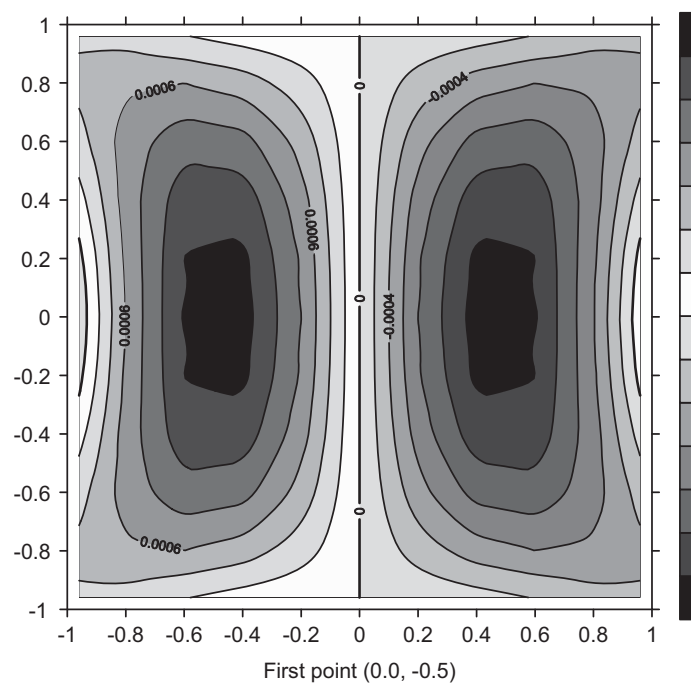

C

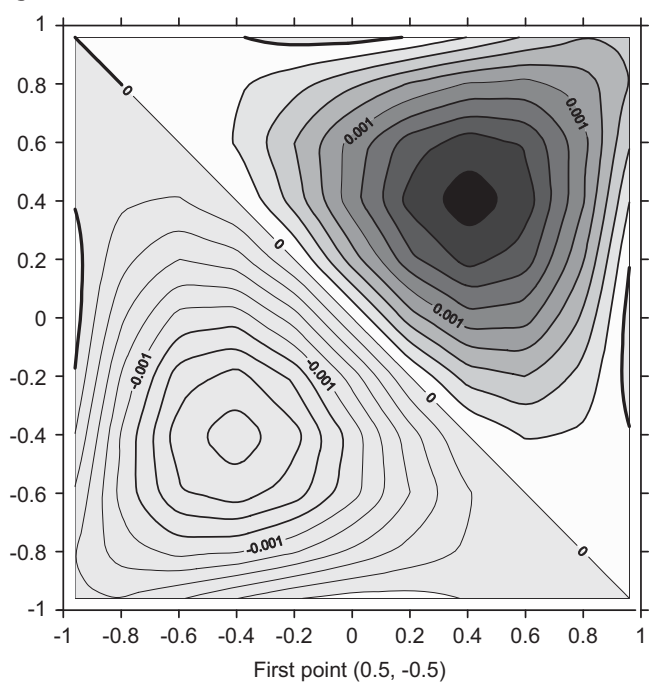

e



b
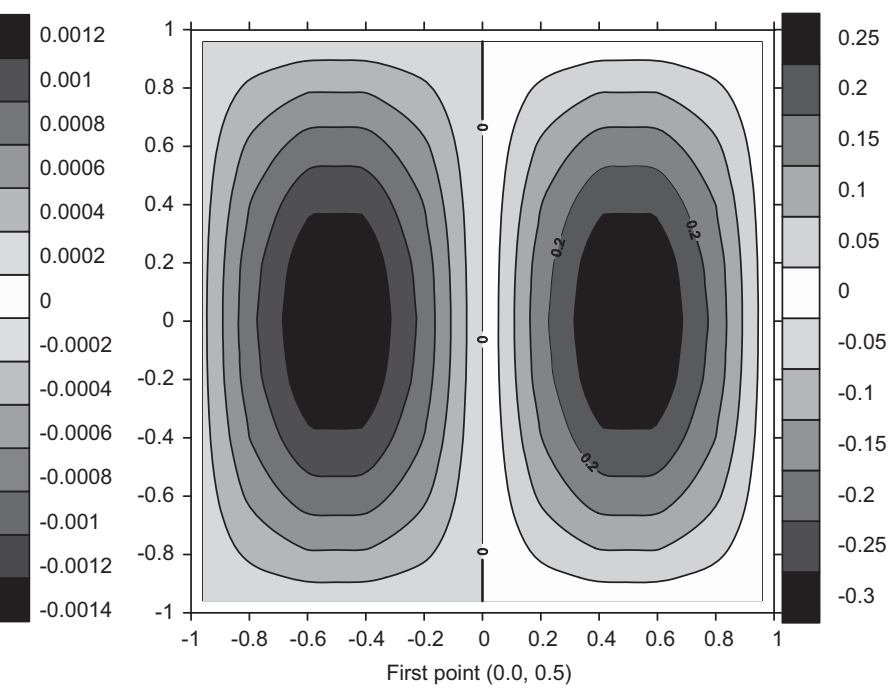

d

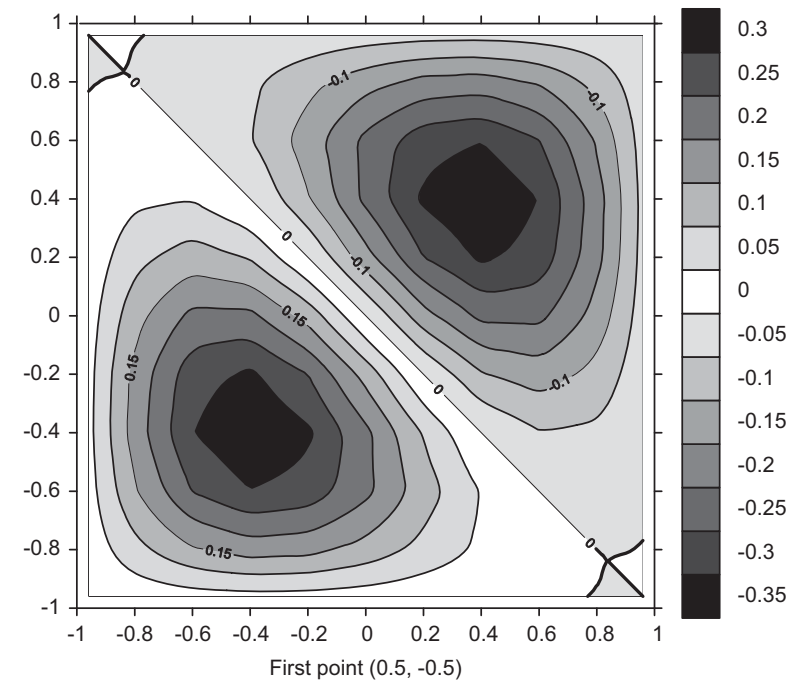

$f$


Fig. 13. (a), (c) and (e) The real-part determinant for the fictitious frequency with multiplicity of two $(P=2$ ), $R=3.51$. (b), (d) and (f) The imaginary-part determinant for the fictitious frequency with multiplicity of two $(P=2), R=3.51$. 


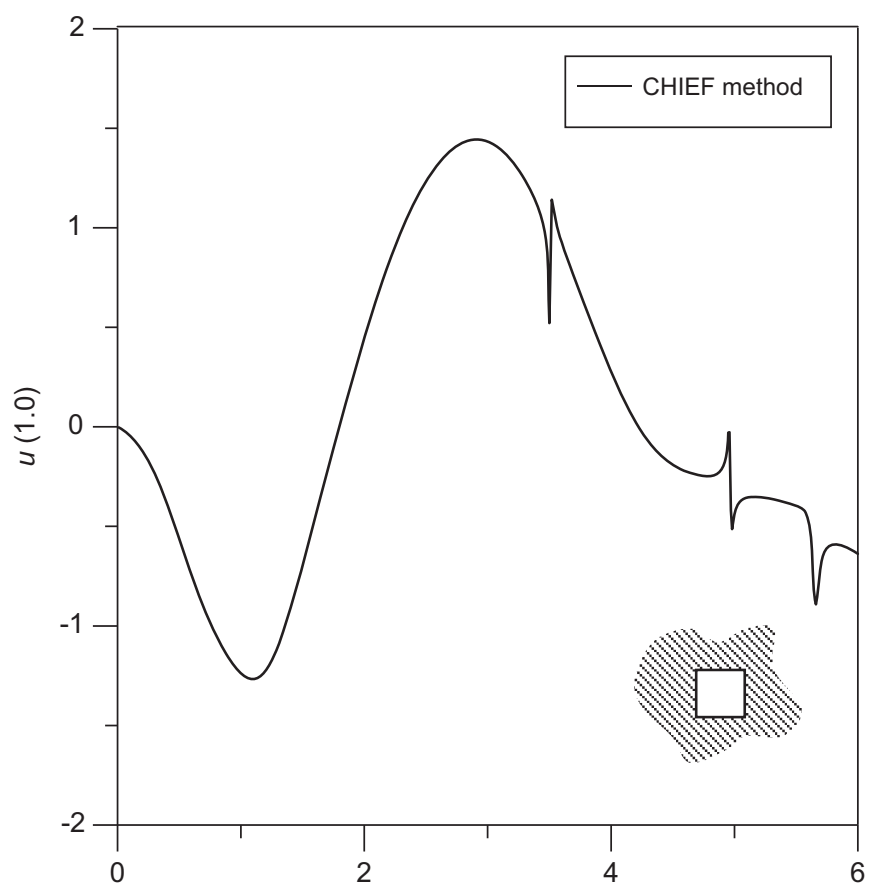

Fig. 14. The boundary value versus the wave number $k$ by locating two CHIEF points on the nodal line.



Fig. 15. The boundary value versus the wave number $k$ by locating two CHIEF points away from the nodal line.

techniques are employed to detect the fictitious frequencies as shown in Fig. 9. Forty boundary elements are considered.

1. Multiplicity of one $(k=2.22)$ : For selecting all the possible CHIEF points, their positions locate inside the square section as shown in Fig. 10. In this case, the determinants of Eq. (49) were calculated for each interior point and were plotted as shown in Fig. 11(a) and (b). Contour plot shows the distribution of the magnitude of the real and imaginary parts of determinant. The selected CHIEF point of the dark color is valider than the point with the white color. The failure points are found on the nodal line labeled by zero value. Without locating any CHIEF points, all fictitious frequencies appear as shown in Fig. 12(a). If one proper CHIEF point is selected, the fictitious frequency $(k=2.22)$ is suppressed as shown in Fig. 12(b).

2. Multiplicity of two $(k=3.51)$ : In this case, the first CHIEF point was fixed at either $(0,0.5)$ or $(-0.5,-0.5)$, and then consider the other CHIEF point as a variable. By selecting all the possible CHIEF points, their positions locate inside the square section as shown in Fig. 10. The value of determinants were calculated for each interior point by changing the second CHIEF point and were plotted in Fig. 13(a)-(f). Contour plot shows the distribution of the magnitude of the real and imaginary parts of determinants. The selected CHIEF point of the dark color is valider than the point with the white color. The failure points are found on the nodal line labeled by zero value. If the two selected points locate on the nodal line, the fictitious frequency $(k=3.51)$ cannot be suppressed as shown in Fig. 14. By selecting the two valid CHIEF points and locating them away from the nodal line, the fictitious frequencies disappear as shown in Fig. 15.

\section{Conclusions}

The fictitious frequency in BEM was revisited. In order to overcome the rank-deficiency problem due to fictitious frequency, the CHIEF method was reformulated in a unified manner by using the Fredholm alternative theorem and SVD updating technique in a discrete system for arbitrary-shape problems. The fictitious mode and frequency were simultaneously obtained in the left singular vectors of SVD once the updating matrix was decomposed by using the SVD technique. Mathematical structures for the four influence matrices in the dual BEM were found by using the SVD. Besides, the minimum number of valid CHIEF points was also addressed. A criterion for checking the number and position of the CHIEF points was analytically derived in the discrete system by using unitary vectors. In addition, the source of numerical instability due to fictitious frequencies was found to originate from the term of zero divided by zero. Numerical examples of the cylinder and square radiator sections were demonstrated to see the validity of the unified formulation.

\section{Acknowledgments}

Financial support from the National Science Council under Grant no. NSC-97-2211-E-019-015 for National Taiwan Ocean University is gratefully acknowledged. The Wu Ta-You award to the first author is also highly appreciated.

\section{References}

[1] Burton AJ, Miller GF. The application of integral equation methods to numerical solutions of some exterior boundary value problem. Proceedings of the Royal Society of London. Series A 1971;323:201-10.

[2] Schenck HA. Improved integral formulation for acoustic radiation problems. Journal of Acoustical Society of America 1976;44(1):41-58.

[3] Chen JT, Liu LW, Hong HK. Spurious and true eigensolutions of Helmholtz BIEs and BEMs for a multiply-connected problem. Proceedings of the Royal Society of London. Series A 2003;459(2036):1891-924.

[4] Benthien GW, Schenck HA. Nonexistence and nonuniqueness problems associated with integral equation method in acoustic. Computers \& Structures 1997;65:295-305. 
[5] Chen JT, Chen IL, Chen KH. Treatment of rank-deficiency in acoustics using SVD. Journal of Computational Acoustics 2006;14(2):157-83.

[6] Juhl PA Numerical study of the coefficient matrix of the boundary element method near characteristic frequencies. Journal of Sound and Vibration 1994; 175(1):39-50.

[7] Poulin S. A boundary element model for diffraction of water waves on varying water depth. PhD dissertation, Department of Hydrodynamics and Water Resources, Technical University of Denmark, ISVA Series Paper no. 64, ISSN 0107-1092, Lyngby; 1997.
[8] Chen IL, Chen JT, Kuo SR, Liang MT. A new method for true and spurious eigensolutions of arbitrary cavities using the combined Helmholtz exterior integral equation method. Journal of Acoustical of Society America 2001;109(3):982-98.

[9] Chen IL, Chen JT, Liang M. Analytical study and numerical experiments for radiation and scattering problems using the CHIEF method. Journal of Sound and Vibration 2000;248(5):809-28.

[10] Wu TW, Lobitz DW. SuperCHIEF: a modified CHIEF method. SANDIA Labs Report SAND-90-1266; 1991. 